\title{
Monte Carlo simulation of the SABRE PoP background
}

M. Antonello ${ }^{a}$, E. Barberio ${ }^{\text {b }}$, T. Baroncelli ${ }^{b}$, J. Benziger ${ }^{c}$, L. J. Bignell ${ }^{\text {d }}$, I. Bolognino ${ }^{\mathrm{a}, \mathrm{e}}$, F. Calaprice ${ }^{\mathrm{f}}$,

S. Copellog,h, D. D'Angelo ${ }^{\text {a,e }}$, G. D'Imperio ${ }^{\mathrm{i}}$, I. Dafinei ${ }^{\mathrm{i}}$, G. Di Carlo ${ }^{\mathrm{g}}$, M. Diemoz ${ }^{\mathrm{i}}$, A. Di Ludovico ${ }^{\mathrm{f}}$,

A. R. Duffy ${ }^{\mathrm{j}, \mathrm{k}}$, F. Froborg ${ }^{\mathrm{l}}$, G. K. Giovanetti ${ }^{\mathrm{f}}$, E. Hoppe ${ }^{\mathrm{m}}$, A. Ianni ${ }^{\mathrm{g}}$, L. Ioannucci ${ }^{\mathrm{g}}$, S. Krishnan ${ }^{\mathrm{k}}$,

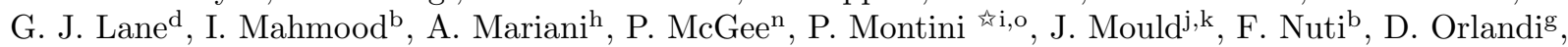

M. Paris ${ }^{b, g}$, V. Pettinacci ${ }^{i}$, L. Pietrofaccia ${ }^{\mathrm{f}}$, D. Prokopovich ${ }^{\mathrm{p}}$, S. Rahatlou ${ }^{\mathrm{i}, \mathrm{o}}$, N. Rossi ${ }^{\mathrm{i}}$, A. Sarbutt ${ }^{\mathrm{p}}$,

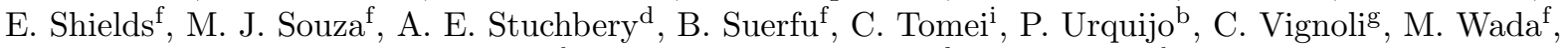
A. Wallner ${ }^{\mathrm{d}}$, A. G. Williams ${ }^{\mathrm{n}}$, J. Xu$^{\mathrm{f}}$, M. Zurowski ${ }^{\mathrm{b}}$

The SABRE Collaboration

${ }^{a}$ INFN - Sezione di Milano, Milano I-20133, Italy

${ }^{b}$ School of Physics, The University of Melbourne, Melbourne, VIC 3010, Australia

${ }^{c}$ Chemical Engineering Department, Princeton University, Princeton, NJ 08544, USA

${ }^{d}$ Department of Nuclear Physics, The Australian National University, Canberra, ACT 2601, Australia

${ }^{e}$ Dipartimento di Fisica, Università degli Studi di Milano, Milano I-20133, Italy

${ }_{f}$ Physics Department, Princeton University, Princeton, NJ 08544, USA

${ }^{g}$ INFN - Laboratori Nazionali del Gran Sasso, Assergi (L'Aquila) I-67100, Italy

${ }^{h}$ INFN - Gran Sasso Science Institute, L'Aquila I-67100, Italy

${ }^{i}$ INFN - Sezione di Roma, Roma I-00185, Italy

${ }^{j}$ ARC Centre of Excellence for All-Sky Astrophysics (CAASTRO), Australia

${ }^{k}$ Centre for Astrophysics and Supercomputing, Swinburne University of Technology, PO Box 218, Hawthorn, Victoria 3122, Australia

${ }^{l}$ Imperial College London, High Energy Physics, Blackett Laboratory, London SW7 2BZ, United Kingdom

${ }^{m}$ Pacific Northwest National Laboratory, y, 902 Battelle Boulevard., Richland, WA 99352, USA

${ }^{n}$ The University of Adelaide, Adelaide, South Australia, 5005 Australia

${ }^{\circ}$ Dipartimento di Fisica, Sapienza Università di Roma, Roma I-00185, Italy

${ }^{p}$ Australian Nuclear Science and Technology Organization, Lucas Heights, NSW 2234, Australia

\begin{abstract}
SABRE (Sodium-iodide with Active Background REjection) is a direct dark matter search experiment based on an array of radio-pure $\mathrm{NaI}(\mathrm{Tl})$ crystals surrounded by a liquid scintillator veto. Twin SABRE experiments in the Northern and Southern Hemispheres will differentiate a dark matter signal from seasonal and local effects. The experiment is currently in a Proof-of-Principle ( $\mathrm{PoP})$ phase, whose goal is to demonstrate that the background rate is low enough to carry out an independent search for a dark matter signal, with sufficient sensitivity to confirm or refute the DAMA result during the following full-scale experimental phase. The impact of background radiation from the detector materials and the experimental site needs to be carefully investigated, including both intrinsic and cosmogenically activated radioactivity. Based on the best knowledge of the most relevant sources of background, we have performed a detailed Monte Carlo study evaluating the expected background in the dark matter search spectral region. The simulation model described in this paper guides the design of the full-scale experiment and will be fundamental for the interpretation of the measured background and hence for the extraction of a possible dark matter signal.
\end{abstract}

Keywords:

SABRE, WIMP, dark matter, annual modulation, $\mathrm{NaI}(\mathrm{Tl})$

\section{Introduction}

¿ Currently at Dipartimento di Fisica Università di Roma Tre I-00146 Roma and INFN Sezione di Roma Tre - 00146, Italy

Preprint submitted to Astroparticle Physics
The existence of dark matter has been inferred from varied astrophysical techniques [1, 2, 3]. Sev- 
eral hypotheses have been formulated about the nature of dark matter, with Weakly Interacting Massive Particles (WIMPs) distributed in the galactic halo one of the most promising candidates [4, 5]. A number of experiments, operated worldwide in underground laboratories, have been searching for many years for direct signals of dark matter interactions, mostly focusing on nuclear recoils produced by WIMP collisions. While most experiments with rapidly increasing sensitivity report a null observation, an observation compatible with dark matter interaction in the DAMA [6] detector remains unverified after almost two decades.

The dark matter signal in an Earth-based detector is expected to modulate yearly due to the change of the Earth's velocity relative to the galactic halo, produced by the orbital motion of the Earth around the Sun. The long-standing result from DAMA, an experiment comprised of a 250-kg array of highly-pure $\mathrm{NaI}(\mathrm{Tl})$ crystals at the Gran Sasso National Laboratory (LNGS), is consistent with this scenario. The modulation is observed with a robust $9.3 \sigma$ statistical significance, and a phase compatible with the one expected for the dark matter modulation. Recently, the DAMA collaboration has released the first results from their Phase-2 experiment [7, confirming the evidence of a signal that meets all the requirements of a modelindependent dark matter annual modulation signature at $12.9 \sigma$ significance. When interpreted in the standard WIMP framework, the results from DAMA are in conflict with other results from experiments using different target materials [8, 9, 10, 11. However such comparison is based on several assumptions of the astrophysical and nuclear models, and on the nature of dark matter particles and their interaction. A model-independent test of the DAMA results is best achieved with an experiment which uses the same target material and detection technique. The Sodium-iodide with Active Background REjection (SABRE) experiment [12, 13] is designed for this purpose and focuses on the achievement of a very low background: the $\mathrm{NaI}(\mathrm{Tl})$ crystals, the photosensors and all detector materials are designed for ultra-high radiopurity; in addition, active rejection of the residual background is obtained with a liquid scintillator veto.

Presently, two arrays of $\mathrm{NaI}(\mathrm{Tl})$ crystals: the COSINE-100 experiment [14] at the YangYang Laboratory in South Korea and the ANAIS experiment [15] at the Canfranc Laboratory in Spain, are in a data taking phase, with a background level 2-3 times higher than DAMA. Their results, even after several years of operation, might not resolve all possible scenarios in interpreting the DAMA signal as a dark matter signature.

The SABRE project foresees the installation of twin detectors at LNGS (Italy) and SUPL (Stawell Underground Physics Laboratory), an underground site in Australia. This unique combination of two high sensitivity $\mathrm{NaI}(\mathrm{Tl})$ detectors will be of great interest for dark matter research through annual modulation, beyond the goal of confirming or refuting DAMA. In fact, the dual site will provide for the first time an effective way to identify any possible season-related contribution to an observed modulation, thanks to the phase inversion between the two hemispheres.

The first phase of the SABRE experiment is the so-called Proof-of-Principle ( $\mathrm{PoP})$ phase and will take place at LNGS during 2018. The goal of the PoP phase is to demonstrate with a high-purity crystal operated inside a liquid scintillator veto that backgrounds are in fact low enough to carry out a reliable test of the DAMA result in the full-scale experiment.

The sensitivity for suitably well-shielded experiments undertaking direct dark matter searches is generally limited by the background rate induced by radioactive contaminants in the detector material and in the materials used for the construction of the experimental setup. Such radioactive contamination may come from long-lived, naturallyoccurring isotopes or from cosmogenic activation. Careful selection or development of radiopure materials and equipment is mandatory, as well as a very good knowledge of the residual radioactivity.

In this paper, we present a simulation study that evaluates the expected background of the SABREPoP, based on the current knowledge of the most relevant sources. The comparison of the simulation result with the future measurements will allow a full characterization of the detector background and provide important knowledge for the realisation of the full-scale experiment.

\section{The SABRE-PoP design and Monte Carlo simulation}

The PoP setup consists of a crystal detector module, installed inside a cylindrical stainless steel vessel filled with liquid scintillator working as a veto against events with multiple interactions within the 
detector or from outside. The steel vessel is protected from the radioactivity in the experimental hall by a hybrid external shield made of lead, polyethylene and water. The general design of the PoP experimental setup and of its sub-parts is described in [16]. Below we point out the main details and how they are implemented in the simulation, which uses the GEANT4 package [17, 18, version 10.02 patch 3 . The crystal detector module consists of a $\mathrm{NaI}(\mathrm{Tl})$ cylindrical crystal, with diameter 3.7 inches, length 8 inches, and mass $5.2 \mathrm{~kg}$, coupled to two 3-inch diameter photomultiplier tubes (PMTs). The crystal is wrapped in a $200 \mu \mathrm{m}$ thick Polytetrafluoroethylene (PTFE) foil. The 3" Hamamatsu R11065-20 PMTs are described by a simplified geometry, made of a quartz window, a Kovar body and a ceramic feedthrough plate, and directly coupled to the crystal. The whole assembly is placed inside a $2 \mathrm{~mm}$-thick cylindrical copper enclosure and held in position by PTFE holders and copper support rods. The external dimensions of the enclosure are $58 \mathrm{~cm}$ length and $14.6 \mathrm{~cm}$ diameter. A view of the internal parts of the enclosure, as implemented in simulation, is shown in Fig. 1 (a).

The liquid scintillator veto consists of a cylindrical stainless steel vessel of $130 \mathrm{~cm}$ diameter $\times 150 \mathrm{~cm}$ length, filled with $\sim 2$ tons of pseudocumene and viewed by ten 8" Hamamatsu R5912 PMTs. The PMTs are described by a simplified geometry: a photocathode ellipsoid plus a cylindrical body made of borosilicate glass, as shown in Fig. 1(b). The mass of each PMT is about $1.1 \mathrm{~kg}$. The crystal enclosure is inserted in a $0.2 \mathrm{~cm}$ thick cylindrical copper tube (16 cm diameter, $121 \mathrm{~cm}$ height) and connected to the top flange of the veto vessel through a stainless steel bar. The liquid scintillator (LS) veto and the Crystal Insertion System (CIS) are shown in Fig. 1(c). The external shielding is made of thick polyethylene (PE) slabs, arranged around the vessel on four sides. The compact polyethylene structure, whose thickness is $10 \mathrm{~cm}$ on the top and $40 \mathrm{~cm}$ on three sides, sustains the weight of a $2 \mathrm{~cm}$ steel plate and of water tanks placed on the top, for a total water thickness of $80 \mathrm{~cm}$. The volume is closed by a polyethylene door $66 \mathrm{~cm}$ thick. All four polyethylene sides are surrounded by water tanks of $91 \mathrm{~cm}$ thickness. The bottom is further shielded by $10 \mathrm{~cm}$ of polyethylene and $15 \mathrm{~cm}$ of lead. The full setup, as implemented in the simulation, is shown in Fig. 1(d).

For the SABRE simulation, we have chosen the Shielding physics list recommended for under-

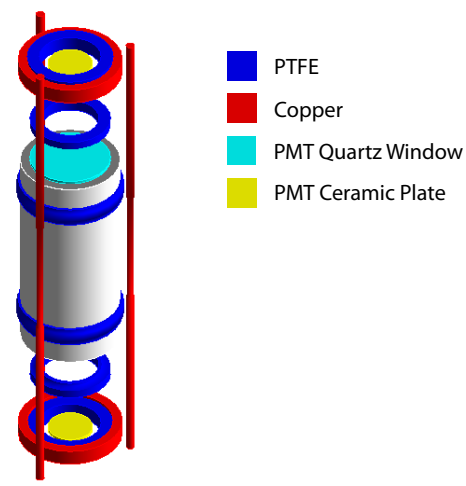

(a) Detector Module (internal parts $\mathrm{NaI}(\mathrm{Tl})$ crystal in grey).

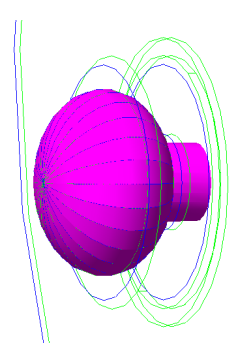

(b) Veto PMT

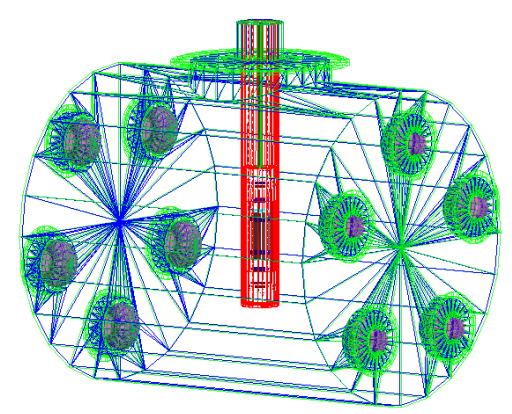

(c) Steel vessel and Crystal Insertion System (CIS)

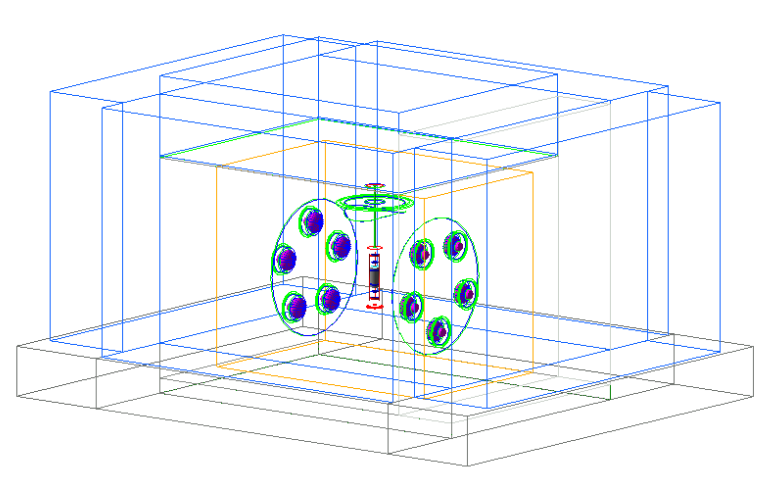

(d) Full setup including external shielding

3 Figure 1: The SABRE-PoP setup as modeled and rendered in Geant4. 
ground low-background experiments, with the addition of the GEANT4 "option 4" for the electromagnetic (EM) part [19]. The package for EM interactions includes the Wentzel VI model at high energy, Msc95 model below $100 \mathrm{MeV}$ 20, photon models from Livermore and Penelope, and Livermore ionisation model for electrons [21, 22. The hadronic interaction model includes elastic, inelastic, capture and fission processes; precision models are used for neutrons with energy below $20 \mathrm{MeV}$. The production and transport of optical photons both in crystal and in the LS veto have not been included in the simulation results described here, however, their inclusion is being pursued currently.

\section{Radioactive contamination of the SABRE-PoP materials}

The most relevant sources of radioactive contamination in the materials are primordial radionuclides $\left({ }^{238} \mathrm{U},{ }^{232} \mathrm{Th}\right.$ and their daughters and ${ }^{40} \mathrm{~K}$ ), anthropogenic radionuclides (e.g. ${ }^{137} \mathrm{Cs}$ ), cosmogenic radionuclides and environmental radioactive noble gases, such as ${ }^{222} \mathrm{Rn}$ and ${ }^{220} \mathrm{Rn}$. The contamination levels of the materials composing the SABRE experiment are based on screening techniques such as gamma ray spectroscopy using High-Purity Ge (HPGe) detectors, neutron activation analysis (NAA), Accelerator Mass Spectroscopy (AMS), and Inductively Coupled Plasma Mass Spectroscopy (ICP-MS).

In sections 3.1 3.4 we list the radioactive contamination of materials used as input to our Monte Carlo simulation. In some cases when the contamination level was below the sensitivity of the measurement, we consider the latter as an upper limit and conservatively use it in the simulation. Secular equilibrium in the $\mathrm{U}$ and $\mathrm{Th}$ decay chains is assumed, unless otherwise specified.

A summary of the experimental components implemented in the GEANT4 simulation with the corresponding materials and masses is reported in Table 1 .

\section{1. $\mathrm{NaI}(\mathrm{Tl})$ Crystals}

Contamination levels for the $\mathrm{NaI}(\mathrm{Tl})$ crystal in the simulation are taken either from measurements performed on the high-purity Astro Grade powder, or from the measurement of a $\sim 2-\mathrm{kg}$ test crystal grown by RMD for the SABRE collaboration in 2015 [16. Uranium and Thorium contamination

\begin{tabular}{|l|l|l|}
\hline Volume Name & Material & Mass $[\mathrm{kg}]$ \\
\hline Crystal & NaI & 5.2 \\
\hline Crystal & PTFE & $2.6 \cdot 10^{-3}$ \\
\hline Enclosure & Copper & $1.5 \cdot 10^{1}$ \\
\hline Crystal Wrapping & 1.2 \\
Enclosure body & Copper & $3.2 \cdot 10^{-1}$ \\
Enclosure small parts & PTFE & $6.0 \cdot 10^{-2}$ \\
Enclosure small parts & Quartz \\
\hline Crystal PMTs & Kovar & $3.1 \cdot 10^{-1}$ \\
\hline Window (x2) & Ceramic \\
Body (x2) & Copper & $1.2 \cdot 10^{1}$ \\
Feedthrough Plate (x2) & $8.3 \cdot 10^{-1}$ \\
\hline Crystal Insertion System (CIS) \\
\hline CIS Tube & Stainless steel \\
CIS Bar & Pseudocumene & $1.9 \cdot 10^{3}$ \\
\hline Veto & Stainless steel & $6.7 \cdot 10^{2}$ \\
\hline Scintillator & Borosilicate glass & $1.1 \cdot 10^{1}$ \\
Vessel & PE \\
PMTs (x10) & PE & $11.5 \cdot 10^{3}$ \\
\hline Shielding & Stainless steel & $1.2 \cdot 10^{3}$ \\
\hline Walls and Top & Pb & $15.6 \cdot 10^{3}$ \\
Base & Water \\
Top & $3.9 \cdot 10^{4}$ \\
Base & Walls and Top & \\
\hline
\end{tabular}

Table 1: Experimental components implemented in the GEANT4 code with corresponding materials and masses.

values in the powder were found below 1 ppt 23 and showed no increase in the test crystal with respect to the starting powder. The average ${ }^{39} \mathrm{~K}$ level found in the crystal at different positions with two different methods was $9 \pm 1 \mathrm{ppb}[23,24,25]$. The concentration of ${ }^{87} \mathrm{Rb}$, which is another common long-living contaminant in $\mathrm{NaI}$ powder, is below the detection limit of $0.1 \mathrm{ppb}$ in the grown crystal 25. Concerning the isotope ${ }^{210} \mathrm{~Pb}$, this cannot be measured at the low levels required at present, although AMS methods are being developed. We rely on contamination levels measured by other $\mathrm{NaI}$ experiments, through the operation of their grown crystals as scintillators. The DAMA [26] experiment finds that a background contribution from internal ${ }^{210} \mathrm{~Pb}$ is visible only in some of the crystals and quotes a range of activity levels of 0.005 - $0.03 \mathrm{mBq} / \mathrm{kg}$. On the other hand, the ANAIS and COSINE experiments, who both have published their background models and compared with data [27, 28, quote a much larger contamination, around $0.7 \mathrm{mBq} / \mathrm{kg}$ in their best crystals and up to $3.2 \mathrm{mBq} / \mathrm{kg}$. In this work, we have used 0.03 $\mathrm{mBq} / \mathrm{kg}$, the highest level among the ${ }^{210} \mathrm{~Pb}$ contaminations found in DAMA crystals, as we are confident that the SABRE procedure for crystal growth and handling will minimize the ${ }^{210} \mathrm{~Pb}$ contamina- 
tion. A quantitative discussion on the impact of an higher ${ }^{210} \mathrm{~Pb}$ contamination on the SABRE background is given in Section 4, where we report the expected background per $\mathrm{Bq} / \mathrm{kg}$ of ${ }^{210} \mathrm{~Pb}$ activity. Cosmogenic-induced contaminations in the crystals are also relevant for a dark matter detector. Among these is ${ }^{3} \mathrm{H}$, especially worrisome as it decays beta releasing $18.6 \mathrm{keV}$ of energy. The COSINE experiment determines a ${ }^{3} \mathrm{H}$ activity in its $\mathrm{NaI}$ crystals at the level of $0.1 \mathrm{mBq} / \mathrm{kg} 28$. Contaminations of tritium are difficult to predict prior to data taking. They indeed depend strongly on the history of the crystal and cannot be measured with HPGe. The cosmogenic activation in NaI crystals has been extensively studied by the ANAIS collaboration [29]. We considered the list of cosmogenic activated isotopes produced in their $\mathrm{NaI}(\mathrm{Tl})$ crystals, and report their expected activities in Table 2, together with the corresponding half lives. We used the ACTIVIA [30] simulation software to calculate the cosmogenic activation of these isotopes at sea level and during transport by plane, assuming an exposure at sea level of about 1 year plus a transport by plane from USA to Italy ( $\sim 10$ hours of flight). We take into account a corrective factor for the geomagnetic effect. The resulting values, reported in Table 2, have been used as input to our simulations. For ${ }^{22} \mathrm{Na}$ only, we used the value measured with HPGe at LNGS on Astro Grade powder 31, which is higher than the value simulated with ACTIVIA. For the long-lived ${ }^{129} \mathrm{I}$, which is treated as stable by ACTIVIA (15.7 million years half life), we have used the value measured by DAMA [26] in their crystals. When our own measurements of cosmogenic activation in the SABRE-PoP crystal operated as a scintillator will become available, we will use the simulated spectra with the correct activities to accurately model the cosmogenic background.

\subsection{Crystal PMTs and reflector foil}

The radioactivity values of PMTs are based on measurements by the XENON Collaboration. They have performed extensive HPGe screening of the Hamamatsu PMT R11410 which is identical to the model R11065 except for the photocathode material. Assuming no significant radioactivity can be attributed to the tiny amount of photocathode material, we have adopted the measurements (or upper limits) from 32 in our simulation. The screening was performed on the main raw materials constituting the tubes and on batches of assembled PMTs.

\begin{tabular}{|c|c|c|c|}
\hline \multicolumn{4}{|c|}{ Intrinsic } \\
\hline Isotope & \multicolumn{2}{|c|}{ Activity $[\mathrm{mBq} / \mathrm{kg}]$} & Ref. \\
\hline${ }^{40} \mathrm{~K}$ & \multicolumn{2}{|c|}{0.31} & 16 \\
\hline${ }^{238} \mathrm{U}$ & \multicolumn{2}{|c|}{$<1.2 \cdot 10^{-2}$} & 16 \\
\hline${ }^{232} \mathrm{Th}$ & \multicolumn{2}{|c|}{$<4.1 \cdot 10^{-3}$} & 16 \\
\hline${ }^{87} \mathrm{Rb}$ & \multicolumn{2}{|c|}{$<8.9 \cdot 10^{-2}$} & 16 \\
\hline${ }^{210} \mathrm{~Pb}$ & \multicolumn{2}{|c|}{$<3.0 \cdot 10^{-2}$} & 26 \\
\hline${ }^{85} \mathrm{Kr}$ & \multicolumn{2}{|c|}{$<1.0 \cdot 10^{-2}$} & 26 \\
\hline \multicolumn{4}{|c|}{ Cosmogenic } \\
\hline Isotope & Activity $[\mathrm{mBq} / \mathrm{kg}]$ & Half life [days] & Ref. \\
\hline${ }^{3} \mathrm{H}$ & $1.810^{-2}$ & 4503 & 30 \\
\hline${ }^{22} \mathrm{Na}$ & 0.48 & 949 & 31 \\
\hline${ }^{126} \mathrm{I}$ & 4.1 & 13 & 30 \\
\hline${ }^{129} \mathrm{I}$ & 0.57 & - & 26 \\
\hline${ }^{113} \mathrm{Sn}$ & $9.610^{-2}$ & 115 & 30 \\
\hline${ }^{125} \mathrm{I}$ & 1.9 & 59 & 30 \\
\hline${ }^{121 m} \mathrm{Te}$ & 0.50 & 154 & 30 \\
\hline${ }^{123 m} \mathrm{Te}$ & 0.31 & 119 & 30 \\
\hline${ }^{125 m} \mathrm{Te}$ & 0.69 & 57 & 30 \\
\hline${ }^{127 m} \mathrm{Te}$ & 0.50 & 107 & 30 \\
\hline
\end{tabular}

Table 2: Radioactivity levels assumed for the $\mathrm{NaI}(\mathrm{Tl})$ SABRE crystals.

The results show that the radioactivity levels of assembled PMTs are not always compatible with the sum of the activities of the raw materials (expressed in $[\mathrm{mBq} / \mathrm{PMT}])$. For example, ${ }^{40} \mathrm{~K}$ and ${ }^{60} \mathrm{Co}$ results were higher by about a factor 6 and 10 in the assembled PMT with respect to the sum of the parts. In our simulations, we model the crystal PMTs as the assembly of three components. These are the three that give the highest contribution in terms of mass and radioactivity, namely the Kovar body, the quartz window and the ceramic feedthrough plates. The contamination values assigned to each of the three components are reported in Table 3 and have been calculated from the values in Table 3 and 4 of [32, divided by the mass of the GEANT4 solid corresponding to the given PMT component ${ }^{1}$. To account for the higher radioactivity levels measured in the assembled PMT, the above values have been rescaled so that, for each isotope, the summed contribution from the three parts matches the total measured value from Table 5 of 32 and at the same time the ratios of activity levels in the three parts are kept constant and equal to those measured in the raw materials.

The contaminations of ${ }^{235} \mathrm{U}$ and ${ }^{137} \mathrm{Cs}$ are also reported in 32, however, since they are upper limits or non-standard contaminations, they have not been considered in this work.

PMTs are coupled to the crystal by using Dow

\footnotetext{
${ }^{1}$ Masses of GEANT4 solids have been compared with Table 2 of the reference and found in agreement.
} 


\begin{tabular}{|c|c|c|c|}
\hline \multirow{2}{*}{ Isotope } & \multicolumn{3}{|c|}{ Activity [mBq/PMT] } \\
\cline { 2 - 4 } & Body & Window & Ceramic plate \\
\hline${ }^{40} \mathrm{~K}$ & $<5.9$ & $<0.48$ & 6.5 \\
${ }^{60} \mathrm{Co}$ & 0.65 & $<0.042$ & $<0.19$ \\
${ }^{238} \mathrm{U}$ & $<0.52$ & $<1.8$ & 13 \\
${ }^{226} \mathrm{Ra}$ & $<0.29$ & 0.040 & 0.29 \\
${ }^{232} \mathrm{Th}$ & $<0.0098$ & $<0.037$ & 0.70 \\
${ }^{228} \mathrm{Th}$ & $<0.41$ & $<0.015$ & 0.13 \\
\hline
\end{tabular}

Table 3: Radioactivity levels of PMT components, obtained by rescaling the values of raw materials from Table 3 and 4 of [32, in order to obtain the same total PMT radioactivity as reported in their Table 5 .

Corning optical silicone grease. Its contribution in the radioactivity background is negligible. This statement is supported by the comparison of Dow Corning radioactivity level 33 with the PMT window. Since both of materials are located close to the crystal they can be compared to each other. The PMT window has a radioactivity level 5 times higher than the optical grease for ${ }^{238} \mathrm{U}, 3$ times higher for ${ }^{232} \mathrm{Th}$ and 27 higher for ${ }^{40} \mathrm{~K}$. Moreover, the mass of the PMT window is greater by about 60 times than the mass of optical grease that will likely be used for the coupling.

To evaluate the background contribution from the reflector material wrapped around the crystal, we assumed the contamination values listed in Table 4. measured by ICP-MS by the XENON experiment on a thin PTFE sheet used as a light reflector 34.

\begin{tabular}{|c|c|}
\hline Isotope & Activity $[\mathrm{mBq} / \mathrm{kg}]$ \\
\hline${ }^{40} \mathrm{~K}$ & 3.1 \\
${ }^{238} \mathrm{U}$ & 0.25 \\
${ }^{232} \mathrm{Th}$ & 0.5 \\
\hline
\end{tabular}

Table 4: Radioactivity levels of PTFE reflector foil 34.

\subsection{Copper and PTFE parts}

The crystal enclosure and the crystal insertion system are mainly made of oxygen-free highthermal-conductivity (OFHC) C10100 copper. The intrinsic background coming from copper parts has been evaluated taking into account the contamination of ${ }^{238} \mathrm{U}$ and ${ }^{232} \mathrm{Th}$ decay chains and ${ }^{40} \mathrm{~K}$. Since the copper used in the manufacturing of the enclosure comes from a batch also used by the CUORE experiment, activities were assumed to be at the same level of those measured by the CUORE collaboration [35. The values are reported in Table 5 . In the background simulation, we have also taken into account the cosmogenic radio-activation of copper. In a study performed for the XENON experiment the cosmogenic activation of OFHC copper was measured after 345 days of exposure to cosmic rays at $3470 \mathrm{~m}$ above sea level and the high-altitude activation measurements were then converted into specific saturation activities at sea level [36. The values are reported in Table 5

\begin{tabular}{|c|c|c|c|}
\hline \multicolumn{4}{|c|}{ Intrinsic } \\
\hline Isotope & \multicolumn{2}{|c|}{ Activity $[\mathrm{mBq} / \mathrm{kg}]$} & Ref. \\
\hline${ }^{40} \mathrm{~K}$ & \multicolumn{2}{|c|}{0.7} & 35 \\
\hline${ }^{238} \mathrm{U}$ & \multicolumn{2}{|c|}{0.065} & 35 \\
\hline${ }^{232} \mathrm{Th}$ & \multicolumn{2}{|c|}{0.002} & 35 \\
\hline \multicolumn{4}{|c|}{ Cosmogenic } \\
\hline Isotope & Activity $[\mathrm{mBq} / \mathrm{kg}]$ & Half life [days] & Ref. \\
\hline${ }^{60} \mathrm{Co}$ & 0.340 & 1925 & 36 \\
\hline${ }^{58} \mathrm{Co}$ & 0.798 & 71 & 36 \\
\hline${ }^{57} \mathrm{Co}$ & 0.519 & 272 & 36 \\
\hline${ }^{56} \mathrm{Co}$ & 0.108 & 77 & 36 \\
\hline${ }^{54} \mathrm{Mn}$ & 0.154 & 312 & 36 \\
\hline${ }^{46} \mathrm{Sc}$ & 0.027 & 84 & 36 \\
\hline${ }^{59} \mathrm{Fe}$ & 0.047 & 44 & 36 \\
\hline${ }^{48} \mathrm{~V}$ & 0.039 & 16 & 36 \\
\hline
\end{tabular}

Table 5: Radioactivity levels assumed for the copper parts of the SABRE crystal enclosure.

Several PTFE rings are used in the crystal enclosure to hold the crystal and the PMTs in place. The XENON collaboration reported on an extensive material screening campaign 34, where no evidence for radioactive contaminants in PTFE was present within the spectrometer sensitivity. We conservatively use these upper limits (Table 6) as activity values in our simulations.

\begin{tabular}{|c|c|}
\hline Isotope & Activity $[\mathrm{mBq} / \mathrm{kg}]$ \\
\hline${ }^{40} \mathrm{~K}$ & $<2.25$ \\
${ }^{238} \mathrm{U}$ & $<0.31$ \\
${ }^{232} \mathrm{Th}$ & $<0.16$ \\
${ }^{60} \mathrm{Co}$ & $<0.11$ \\
${ }^{137} \mathrm{Cs}$ & $<0.13$ \\
\hline
\end{tabular}

Table 6: Radioactivity levels assumed for the PTFE parts of the SABRE crystal enclosure. Values are taken from 34.

\subsection{Veto components: Stainless steel, PMTs and Liquid Scintillator}

Stainless steel is used in the veto vessel and in the crystal insertion system. The SABRE-PoP veto vessel was manufactured by Allegheny Bradford Corporation (ABC) in Bradford, PA, USA. The main components are a $1 / 4$ " thick plate that was rolled into the cylinder and a $3 / 8$ " thick plate that was used for the side walls, the top flange and 
the top plate. Table 7 summarizes the radioactivity of steel samples from Stainless Plate Products (SPPUSA) measured using the glow discharge mass spectrometry (GDMS) method. The most conservative values (higher contamination) have been used for the steel vessel and the CIS steel bar.

\begin{tabular}{|c|c|c|}
\hline \multirow{2}{*}{ Isotope } & \multicolumn{2}{|c|}{ Activity $[\mathrm{mBq} / \mathrm{kg}]$} \\
\cline { 2 - 3 } & $\begin{array}{c}\text { Lot n.S536 } \\
\text { Thickness 3/8" }\end{array}$ & $\begin{array}{c}\text { Lot } \mathrm{n} . \mathrm{T} 915 \\
\text { Thickness 1/4" }\end{array}$ \\
\hline${ }^{40} \mathrm{~K}$ & 0.12 & $<0.03$ \\
${ }^{238} \mathrm{U}$ & 3.7 & 0.49 \\
${ }^{232} \mathrm{Th}$ & $<0.41$ & 0.082 \\
\hline
\end{tabular}

Table 7: Radioactivity inferred from concentration measurements with GDMS of stainless steel used for the SABREPoP vessel and CIS 37.

The active veto uses ten 8" Hamamatsu R5912 PMTs. The PMT is made from low-radioactivity borosilicate glass and the corresponding contaminations (Table 8) have been measured by the DarkSide-50 collaboration, which uses the same model 38.

\begin{tabular}{|c|c|}
\hline Isotope & Activity $[\mathrm{mBq} / \mathrm{PMT}]$ \\
\hline${ }^{40} \mathrm{~K}$ & 649 \\
${ }^{238} \mathrm{U}$ & 883 \\
${ }^{232} \mathrm{Th}$ & 110 \\
${ }^{235} \mathrm{U}$ & 41 \\
\hline
\end{tabular}

Table 8: Radioactivity levels of the Hamamatsu R5912 Veto PMTs. Values are taken from 38.

The SABRE-PoP liquid scintillator veto consists of $\sim 2$ ton of high-purity pseudocumene (PC) doped with $3 \mathrm{~g} / \mathrm{l}$ PPO as wavelength shifter. The scintillator will be provided by the Borexino experimental facilities. Consequently we have adopted the contamination levels measured by Borexino [39, as reported in Table 9

\begin{tabular}{|c|c|}
\hline Isotope & Activity $[\mathrm{mBq} / \mathrm{kg}]$ \\
\hline${ }^{40} \mathrm{~K}$ & $3.5 \cdot 10^{-7}$ \\
${ }^{238} \mathrm{U}$ & $<1.2 \cdot 10^{-6}$ \\
${ }^{232} \mathrm{Th}$ & $<1.2 \cdot 10^{-6}$ \\
${ }^{210} \mathrm{~Pb}$ & $1.7 \cdot 10^{-6}$ \\
${ }^{210} \mathrm{Bi}$ & $1.7 \cdot 10^{-6}$ \\
${ }^{7} \mathrm{Be}$ & $<1.2 \cdot 10^{-6}$ \\
${ }^{14} \mathrm{C}$ & $4.1 \cdot 10^{-1}$ \\
${ }^{39} \mathrm{Ar}$ & $3.5 \cdot 10^{-6}$ \\
${ }^{85} \mathrm{Kr}$ & $3.5 \cdot 10^{-7}$ \\
\hline
\end{tabular}

Table 9: Radioactivity levels assumed for the liquid scintillator. Values are taken from [39].

\subsection{External Shield}

The shielding geometry is implemented in the simulation but is treated only as a passive material, i.e. radioactivity of the shielding and its contribution to the background budget of the PoP is not included in this work. Previous simulations performed with the same Monte Carlo code to support the shielding design show that a radio-purity at the level measured at LNGS, by HPGe spectroscopy on samples of SABRE polyethylene ( $\mathrm{K}$ and gamma emitters from $\mathrm{U} / \mathrm{Th}$ chain), gives a negligible contribution to the total background.

The same is true for the contribution from radiogenic neutrons in the LNGS rock, in the passive shielding materials and in the liquid scintillator. A preliminary estimation of this contribution was performed using the SOURCES 40] code, the results of which were used as an input to the Geant4 simulation.

\section{Results}

We simulated radioactive decays in different components of the setup, according to the radioactive contaminations of the materials described in the previous sections. For every combination of isotope and location inside the setup, we simulated a number of events high enough to keep the statistical uncertainty in the output spectrum well below the percent level for the crystal background and below a few percent for the outermost volumes.

The crystal and the liquid scintillator are treated in the GEANT4 simulation as sensitive detectors, and all the energy deposited by radiated particles inside those volumes is recorded. The optical simulation, meaning the generation, propagation and collection of optical photons from scintillation, is not carried out in this work. We applied a gaussian smearing to the data to account for a $2 \% \cdot \sqrt{E}$ $[\mathrm{MeV}]$ resolution on the reconstructed energy in the $\mathrm{NaI}(\mathrm{Tl})$ crystal, while an energy resolution of $6 \% \cdot \sqrt{E}[\mathrm{MeV}]$ has been considered for the veto signal. These are representative values for detectors such as $\mathrm{NaI}(\mathrm{Tl})$ crystals and Pseudocumene+PPO liquid scintillator detectors [26, 39. The detection efficiency of the liquid scintillator veto is assumed to be $100 \%$ for energy deposited above $100 \mathrm{keV}$ [37]. We evaluated the background contributions in the two distinct operation modes that we anticipate using during the PoP operation: the potassium Measurement Mode (KMM) and the Dark Matter Measurement Mode (DMM), where the liquid 
scintillator detector is used in coincidence or anticoincidence with the crystal, respectively.

\subsection{Expected background in potassium measure- ment mode (KMM)}

The KMM will be used in the PoP to measure the ${ }^{40} \mathrm{~K}$ activity in the crystal, which is a significant background contribution to the energy spectrum in the region of interest for a dark matter search. The electron capture (EC) from potassium decay in the crystal (about 11\% branching ratio) gives an energy deposit around $3.2 \mathrm{keV}$ due to X-ray or Auger de-excitation, in coincidence with a $\gamma$ of $1.46 \mathrm{MeV}$. We assess the sensitivity of the SABREPoP to the measurement of the ${ }^{40} \mathrm{~K}$ activity in the crystal by evaluating the signal-to-background ratio in simulated data. The ${ }^{40} \mathrm{~K}$ signal is defined as an energy deposit between 2 and $4 \mathrm{keV}$ in the crystal ( $1 \sigma$ around the $3 \mathrm{keV}{ }^{40} \mathrm{~K}$ peak) in coincidence with an energy deposit between $1.28 \mathrm{MeV}$ and 1.64 $\mathrm{MeV}$ in the liquid scintillator $(2.5 \sigma$ around the 1.46 $\mathrm{MeV}{ }^{40} \mathrm{~K}$ peak). True coincidences from radioactive sources other than ${ }^{40} \mathrm{~K}$ contaminations in the crystal can mimic the same signature and therefore have to be regarded as background for the potassium measurement.

The background contributions from all the SABRE-PoP components to the crystal energy spectrum in KMM are reported in Fig. 2. Cosmogenic activation is evaluated after 60 days underground. The signal produced by a $10 \mathrm{ppb}^{n a t} K$ contamination in the crystal is superimposed in red in Fig. 2(b) for comparison.

The crystal cosmogenic activation gives the most relevant contribution to the background in this measurement mode. This is mostly due to ${ }^{22} \mathrm{Na}$ contamination, since ${ }^{22} \mathrm{Na}$ emits $1275 \mathrm{keV} \gamma$ rays that often leave a deposit in the energy region selected for potassium. The second-highest contribution to the background in KMM comes from the veto (namely the sum of scintillator, steel vessel and veto PMTs).

As shown in Table 10, the total background is about one order of magnitude lower than the signal given by a $10 \mathrm{ppb}{ }^{\text {nat }} K$ contamination in the crystal, thus demonstrating that the measurement of such potassium level is possible with $\sim 1$ ppb precision in about two months of data taking.
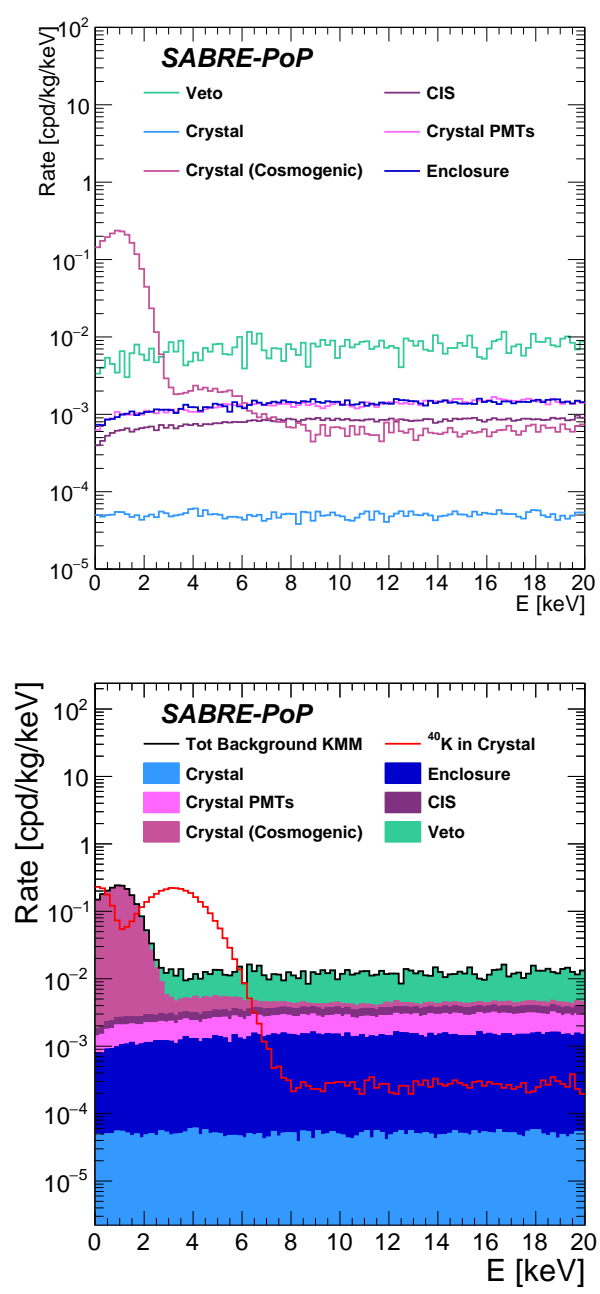

Figure 2: Backgrounds from all SABRE-PoP setup components in a $[0-20] \mathrm{keV}$ region in potassium measurement mode (KMM). The top plot shows the individual contributions from crystal (cosmogenics after 60 days underground and intrinsic backgrounds except ${ }^{40} \mathrm{~K}$ ), phototubes (PMTs), crystal enclosure, insertion system (CIS), and veto (including liquid scintillator, steel vessel and PMTs). The bottom plot shows the stacked backgrounds, and the total (solid black line). The red superimposed line shows the ${ }^{40} \mathrm{~K}$ spectrum from a $10 \mathrm{ppb}{ }^{\text {nat } K}$ contamination in the crystal, which represents the signal in this configuration.

\subsection{Expected background in dark matter measure- ment mode (DMM)}

We estimate the background level attainable by the SABRE-PoP in its planned configuration by looking at energy depositions from 2 to $6 \mathrm{keV}$ in the crystal, in anti-coincidence with the veto. Dark matter interactions are single-site events that give rise to only one energy release. This is not the case for some of the background components, for 


\begin{tabular}{|l|c|}
\hline & $\begin{array}{c}\text { Rate KMM } \\
{[\mathrm{cpd} / \mathrm{kg} / \mathrm{keV}]}\end{array}$ \\
\hline Crystal Cosmogenic & $9.8 \cdot 10^{-3}$ \\
Veto & $6.2 \cdot 10^{-3}$ \\
Enclosure & $1.3 \cdot 10^{-3}$ \\
Crystal PMTs & $1.1 \cdot 10^{-3}$ \\
CIS & $7.7 \cdot 10^{-4}$ \\
Crystal (no $\left.{ }^{40} \mathrm{~K}\right)$ & $5.1 \cdot 10^{-5}$ \\
\hline Total & $2.5 \cdot 10^{-2}$ \\
\hline Crystal ${ }^{40} \mathbf{K}$ & $1.9 \cdot 10^{-1}$ \\
\hline
\end{tabular}

Table 10: Background rates in the region $[2-4] \mathrm{keV}$ in KMM from all the SABRE-PoP setup components, listed in decreasing order. The signal of ${ }^{40} \mathrm{~K}$ in the crystal is reported below the total background and in bold. Cosmogenic backgrounds for crystal, enclosure and CIS are computed after 60 days underground.

example the intrinsic ${ }^{40} \mathrm{~K}$ described above. If the high-energy $1.46 \mathrm{MeV}$ gamma from potassium decay escapes undetected from the crystal volume, the remaining $3.2 \mathrm{keV}$ deposit in the crystal contaminates the low-energy region where a dark matter signal is expected. This can be avoided if the 1.46 $\mathrm{MeV} \gamma$ is detected in the liquid scintillator veto.

The background contributions from all of the SABRE-PoP components to the crystal energy spectrum in DMM is reported in Fig. 3 and the background rates in the region of interest $[2-6] \mathrm{keV}$ are reported in Table 11, highlighting the effect of the liquid scintillator veto. The contribution from cosmogenic activation is evaluated assuming the data taking will start 180 days after the crystal is brought underground. The background from ${ }^{3} \mathrm{H}$ is reported independently as it's the strongest source among cosmogenic isotopes and cannot be vetoed. Apart from this, the most relevant contribution in the $2-6 \mathrm{keV}$ energy region comes from ${ }^{121}$ Te. Despite its short half life $\left(T_{1 / 2}=17\right.$ days $)$, the isotope is regenerated by the presence of the metastable ${ }^{121 m}$ Te $\left(T_{1 / 2}=154\right.$ days $)$ which decays to the ground state with an internal transition probability of 0.886 .

The rejection efficiency of the veto can be evaluated as the ratio of the number of events that give an energy deposition in the crystal in coincidence with a release of at least $100 \mathrm{keV}$ in the liquid scintillator, over the total number of events in the crystal. For the ${ }^{40} \mathrm{~K}$ background in the crystal, in the region of interest for DM search [2-6] keV, the expected veto efficiency is $84 \%$ (see also Fig. 4). Overall the expected background rejection due to the liquid scintillator veto is $44 \%$, which is largely affected by the non-vetoed contribution from ${ }^{3} \mathrm{H}$.
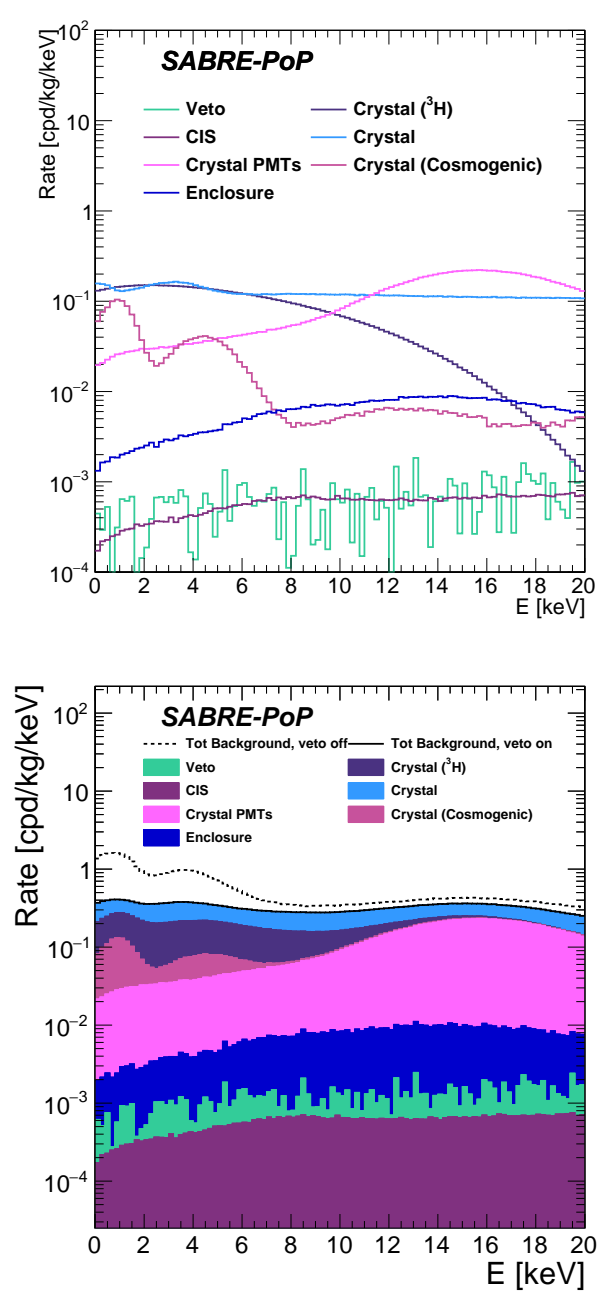

Figure 3: Backgrounds from all SABRE-PoP setup components in a $[0-20] \mathrm{keV}$ region in dark matter measurement mode (DMM). The top plot shows, with veto on, the separate contributions from crystal (intrinsic backgrounds, ${ }^{3} \mathrm{H}$, and cosmogenics after 180 days underground), phototubes (PMTs), crystal enclosure, insertion system (CIS), and veto (including liquid scintillator, steel vessel and PMTs). The bottom plot shows the stacked backgrounds and the total with veto on (solid black line) and veto off (dashed black line).

The simulated radioactive plus cosmogenic activated contamination of the crystal gives the most significant contribution in dark matter measurement mode. This confirms what was already estimated in previous SABRE simulation studies [37. In Fig. 5 the background in a $[0-20] \mathrm{keV}$ region due to the intrinsic and cosmogenic contaminations in the $\mathrm{NaI}(\mathrm{Tl})$ crystal is reported. The background rates in the region of interest $[2-6] \mathrm{keV}$ are re- 


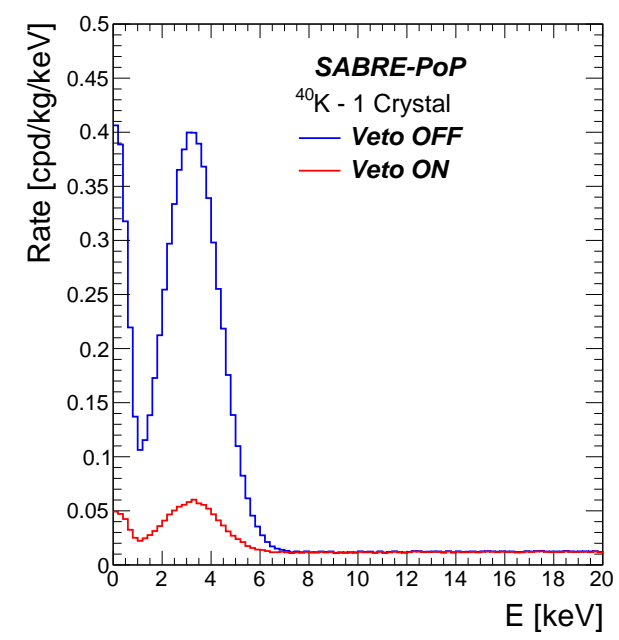

Figure 4: Effect of the LS veto on the ${ }^{40} \mathrm{~K}$ background in the crystal.

\begin{tabular}{|l|c|c|}
\hline & $\begin{array}{c}\text { Rate, veto OFF } \\
{[\mathrm{cpd} / \mathrm{kg} / \mathrm{keV}]}\end{array}$ & $\begin{array}{c}\text { Rate, veto ON } \\
{[\mathrm{cpd} / \mathrm{kg} / \mathrm{keV}]}\end{array}$ \\
\hline Crystal & $3.5 \cdot 10^{-1}$ & $1.5 \cdot 10^{-1}$ \\
Crystal $\left({ }^{3} \mathrm{H}\right)$ & $1.4 \cdot 10^{-1}$ & $1.4 \cdot 10^{-1}$ \\
Crystal Cosmogenic & $2.4 \cdot 10^{-1}$ & $3.1 \cdot 10^{-2}$ \\
Crystal PMTs & $4.3 \cdot 10^{-2}$ & $3.5 \cdot 10^{-2}$ \\
Enclosure & $9.5 \cdot 10^{-3}$ & $3.6 \cdot 10^{-3}$ \\
Veto & $3.0 \cdot 10^{-2}$ & $5.7 \cdot 10^{-4}$ \\
CIS & $3.7 \cdot 10^{-3}$ & $4.6 \cdot 10^{-4}$ \\
\hline Total & $8.2 \cdot 10^{-1}$ & $3.6 \cdot 10^{-1}$ \\
\hline
\end{tabular}

Table 11: Background rate in the region of interest [2 6] keV from all the SABRE-PoP setup components with veto off and on respectively. The contributions are listed in decreasing order with veto on. Cosmogenic backgrounds for crystal, enclosure and CIS are computed after 180 days underground.
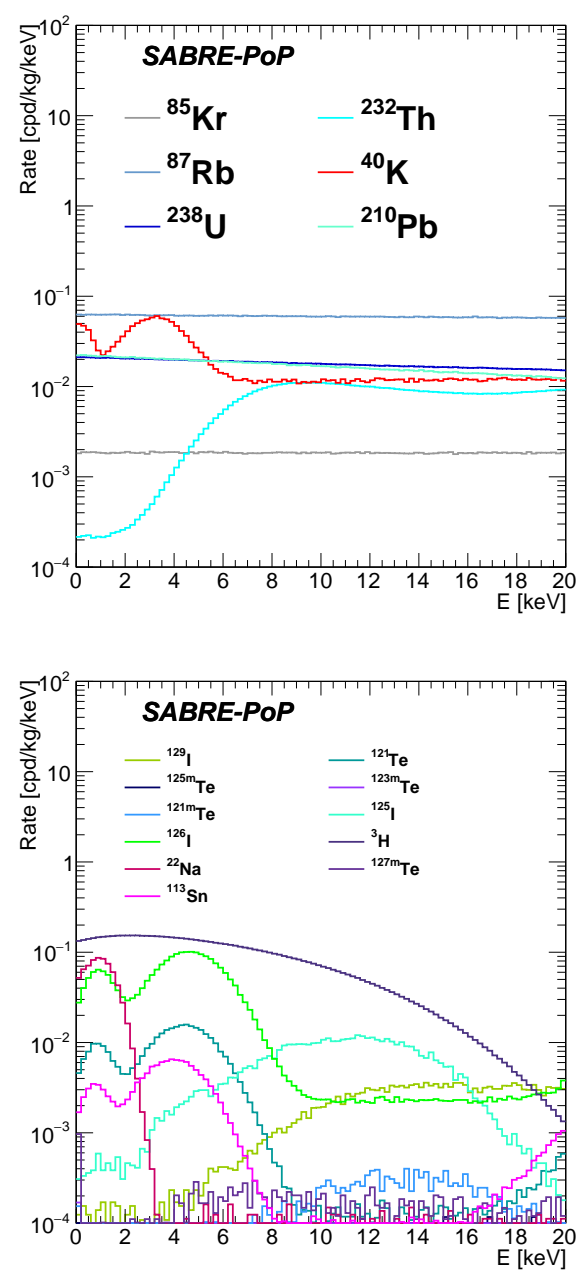

Figure 5: Background in a $[0-20] \mathrm{keV}$ region due to the contaminants in the $\mathrm{NaI}(\mathrm{Tl})$ crystal. Top plot shows the contributions of intrinsic backgrounds. Bottom plot shows the contributions from cosmogenically-activated nuclei after 180 days underground. Veto is applied to the events that release more than $100 \mathrm{keV}$ in the liquid scintillator.

ported in Table 12 .

Among the intrinsic contaminations listed in Table 12 , the most relevant source of background is ${ }^{87} \mathrm{Rb}$, followed by ${ }^{40} \mathrm{~K}$. The ${ }^{87} \mathrm{Rb}$ contamination, however, is assumed equal to the upper limit from the ICPMS measurement. Cosmogenic activation, mainly from ${ }^{3} \mathrm{H}$, has a significant impact on the total background. The total background contribution of this component $\left(1.7 \cdot 10^{-1} \mathrm{cpd} / \mathrm{kg} / \mathrm{keV}\right.$ with veto on) relies on calculations that assume an exposure at sea level of about 1 year plus a transport by plane from USA to Italy, followed by 180 days underground. The ultimate level of cosmogenic ac- 
tivation in the SABRE-PoP crystal will depend on how long the powder and the crystal are exposed to the cosmic ray flux. The results of our simulations highlight that minimising this exposure is crucial. As discussed in Section 3 , the isotopes ${ }^{3} \mathrm{H}$ and ${ }^{210} \mathrm{~Pb}$ contribute significantly to the count rate in the low energy region. Their activity in the SABRE-PoP crystal is difficult to predict at this stage and measuring them is among the goals of the PoP run. From simulations, each $\mu \mathrm{Bq} / \mathrm{kg}$ of ${ }^{3} \mathrm{H}$ activity in the crystal leads to $8.1 \cdot 10^{-3} \mathrm{cpd} / \mathrm{kg} / \mathrm{keV}$ of background. At the same time, we estimate that, for the SABRE-PoP detector, $1 \mu \mathrm{Bq} / \mathrm{kg}$ of ${ }^{210} \mathrm{~Pb}$ will give a rate in the signal region of $0.67 \cdot 10^{-3} \mathrm{cpd} / \mathrm{kg} / \mathrm{keV}$. In the budget of Table 12 we have allowed for a ${ }^{3} \mathrm{H}$ activity of $0.018 \mathrm{mBq} / \mathrm{kg}$ and ${ }^{210} \mathrm{~Pb}$ activity of $0.03 \mathrm{mBq} / \mathrm{kg}$.

\begin{tabular}{|c|c|c|}
\hline Isotope & $\begin{array}{c}\text { Rate, veto OFF } \\
{[\mathrm{cpd} / \mathrm{kg} / \mathrm{keV}]}\end{array}$ & $\begin{array}{c}\text { Rate, veto ON } \\
{[\mathrm{cpd} / \mathrm{kg} / \mathrm{keV}]}\end{array}$ \\
\hline \multicolumn{3}{|c|}{ Intrinsic } \\
\hline${ }^{87} \mathrm{Rb}$ & $6.1 \cdot 10^{-2}$ & $6.1 \cdot 10^{-2}$ \\
\hline${ }^{40} \mathrm{~K}$ & $2.5 \cdot 10^{-1}$ & $4.0 \cdot 10^{-2}$ \\
\hline${ }^{238} \mathrm{U}$ & $2.0 \cdot 10^{-2}$ & $2.0 \cdot 10^{-2}$ \\
\hline${ }^{210} \mathrm{~Pb}$ & $2.0 \cdot 10^{-2}$ & $2.0 \cdot 10^{-2}$ \\
\hline${ }^{85} \mathrm{Kr}$ & $1.9 \cdot 10^{-3}$ & $1.9 \cdot 10^{-3}$ \\
\hline${ }^{232} \mathrm{Th}$ & $1.9 \cdot 10^{-3}$ & $1.7 \cdot 10^{-3}$ \\
\hline Tot Intrinsic & $3.5 \cdot 10^{-1}$ & $1.4 \cdot 10^{-1}$ \\
\hline \multicolumn{3}{|c|}{ Cosmogenic } \\
\hline${ }^{3} \mathrm{H}$ & $1.4 \cdot 10^{-1}$ & $1.4 \cdot 10^{-1}$ \\
\hline${ }^{121} \mathrm{Te}$ & $2.0 \cdot 10^{-1}$ & $2.6 \cdot 10^{-2}$ \\
\hline${ }^{113} \mathrm{Sn}$ & $1.2 \cdot 10^{-2}$ & $2.2 \cdot 10^{-3}$ \\
\hline${ }^{22} \mathrm{Na}$ & $2.1 \cdot 10^{-2}$ & $1.5 \cdot 10^{-3}$ \\
\hline${ }^{125} \mathrm{I}$ & $4.4 \cdot 10^{-4}$ & $4.4 \cdot 10^{-4}$ \\
\hline${ }^{129} \mathrm{I}$ & $1.9 \cdot 10^{-4}$ & $1.9 \cdot 10^{-4}$ \\
\hline${ }^{126} \mathrm{I}$ & $1.8 \cdot 10^{-4}$ & $1.2 \cdot 10^{-4}$ \\
\hline $127 m \mathrm{Te}$ & $6.4 \cdot 10^{-5}$ & $6.4 \cdot 10^{-5}$ \\
\hline $121 m_{\mathrm{Te}}$ & $7.1 \cdot 10^{-5}$ & $3.7 \cdot 10^{-5}$ \\
\hline${ }^{123 m} \mathrm{Te}$ & $1.9 \cdot 10^{-5}$ & $1.3 \cdot 10^{-5}$ \\
\hline${ }^{125 m} \mathrm{Te}$ & $3.8 \cdot 10^{-6}$ & $3.7 \cdot 10^{-6}$ \\
\hline $\begin{array}{c}\text { Tot Cosmogenic } \\
\text { (180 days) }\end{array}$ & $3.8 \cdot 10^{-1}$ & $1.7 \cdot 10^{-1}$ \\
\hline
\end{tabular}

Table 12: Background rate in the region of interest [2-6] keV due to the contaminants in $\mathrm{NaI}(\mathrm{Tl})$ crystals. Both intrinsic and cosmogenically-activated contributions are reported, with veto off and on respectively. The contributions are listed in decreasing order with veto on. The ${ }^{87} \mathrm{Rb}$ contamination is an upper limit from ICPMS measurement. Cosmogenic backgrounds are computed after 180 days underground.

As stated in Sect. 3.5 the background budget due to the shielding materials has not been included in this work, since previous simulations performed with the same Monte Carlo have shown that the contamination of samples of SABRE polyethylene produce a background well below $10^{-3} \mathrm{cpd} / \mathrm{kg} / \mathrm{keV}$ in dark matter measurement mode and therefore can be neglected. A preliminary simulation of radiogenic neutrons in the LNGS rock, in the passive shielding materials and in the liquid scintillator has shown that this contribution is at the level of $10^{-4} \mathrm{cpd} / \mathrm{kg} / \mathrm{keV}$ in dark matter measurement mode.

\section{Conclusions}

The SABRE-PoP operation is expected to start at LNGS in 2018, with the goal of demonstrating that the intrinsic $\mathrm{NaI}(\mathrm{Tl})$ crystal contamination levels and the other backgrounds from the SABRE apparatus itself are low enough to carry out a reliable test of the DAMA result in the projected SABRE full-scale experiment. We evaluated the expected background of the SABRE-PoP with a Monte Carlo simulation based on the current knowledge of the most relevant sources of radioactive contamination. The simulation carefully reproduces the design of the PoP apparatus, with particular attention to the parts close to the crystal detector. We find that the radioactive contamination of the crystal gives the most significant contribution to the low-energy background, confirming the importance in the SABRE strategy of lowering the crystal contamination as much as possible. The radiopurity of the crystals, combined with the active veto technique, allows SABRE to achieve a design background of $0.36 \mathrm{cpd} / \mathrm{kg} / \mathrm{keV}$ in the $2-6 \mathrm{keV}$ energy region, where the maximum amplitude of the modulation was observed by DAMA. It is important to note that this estimation was derived under the assumptions that the intrinsic activities of ${ }^{3} \mathrm{H}$ and ${ }^{210} \mathrm{~Pb}$ are below $0.018 \mathrm{mBq} / \mathrm{kg}$ and $0.03 \mathrm{mBq} / \mathrm{kg}$ respectively. Under these assumptions, the highest contribution to the crystal intrinsic background is ${ }^{87} \mathrm{Rb}$, for which we assumed the upper limit contamination. SABRE aims to be the first $\mathrm{NaI}(\mathrm{Tl})-$ based experiment with a background significantly lower than the one achieved by DAMA. The background model described in this paper will serve as a comparison tool for the interpretation of the measured background and as a guiding tool throughout the design phase of the full scale experiment.

\section{Acknowledgements}

The SABRE program is supported by funding from INFN (Italy), NSF (USA), and ARC 
(Australia, grants LE170100162, LE16010080, DP170101675, LP150100075). F. Froborg has received funding from the European Union's Horizon 2020 research and innovation programme under the Marie Sklodowska-Curie grant agreement No 703650. We acknowledge the generous hospitality and constant support of the Laboratori Nazionali del Gran Sasso (Italy).

\section{References}

[1] P. A. R. Ade, et al., Planck 2015 results. XIII. Cosmological parameters, Astron. Astrophys. 594 (2016) A13. arXiv:1502.01589 doi:10.1051/0004-6361/ 201525830

[2] A. Conley, et al., Supernova Constraints and Systematic Uncertainties from the First 3 Years of the Supernova Legacy Survey, Astrophys. J. Suppl. 192 (2011) 1. arXiv:1104.1443 doi:10.1088/0067-0049/192/1/1

[3] C. Heymans, et al., CFHTLenS tomographic weak lensing cosmological parameter constraints: Mitigating the impact of intrinsic galaxy alignments, Mon. Not. Roy. Astron. Soc. 432 (2013) 2433. arXiv:1303.1808, doi: $10.1093 / \mathrm{mnras} / \mathrm{stt} 601$.

[4] G. Steigman, M. S. Turner, Nuclear Physics B 253 (1985) 375 - 386. doi:https://doi.org/10.1016/ 0550-3213(85) 90537-1

[5] G. Bertone, D. Hooper, J. Silk, Physics Reports 405 (5) (2005) 279 - 390. doi:https://doi.org/10.1016/j. physrep.2004.08.031

[6] Bernabei R. et al. (DAMA/LIBRA Collaboration), Final model independent result of DAMA/LIBRA phase1, European Physical Journal C 73 (2013) 2648. doi:10.1140/epjc/s10052-013-2648-7

[7] R. Bernabei, et al., First model independent results from DAMA/LIBRA-phase2, arXiv: 1805.10486

[8] E. Aprile, et al., First Dark Matter Search Results from the XENON1T Experiment, Phys. Rev. Lett. 119 (2017) 181301.

[9] D. S. Akerib, et al., Results from a Search for Dark Matter in the Complete LUX Exposure, Phys. Rev. Lett. 118 (2017) 021303. doi:10.1103/PhysRevLett. 118.021303

[10] R. Agnese, et al., Search for Low-Mass Weakly Interacting Massive Particles with SuperCDMS, Phys. Rev. Lett. 112 (2014) 241302. doi:10.1103/PhysRevLett. 112.241302

[11] K. Abe, et al., Direct dark matter search by annual modulation with 2.7 years of XMASS-I data, Phys. Rev. D 97 (2018) 102006. doi:10.1103/PhysRevD.97. 102006

[12] J. Xu, et al., SABRE - A test of DAMA with highpurity $\mathrm{NaI}(\mathrm{Tl})$ crystals, AIP Conference Proceedings 1672. doi:http://dx.doi.org/10.1063/1.4927983

[13] F. Froborg, SABRE: WIMP modulation detection in the northern and southern hemisphere, J. Phys. Conf. Ser. 718 (4) (2016) 042021. arXiv:1601.05307 doi: 10.1088/1742-6596/718/4/042021

14] G. Adhikari, et al., Initial performance of the COSINE-100 experiment, The European Physical Journal C 78 (2) (2018) 107. doi:10.1140/epjc/ s10052-018-5590-x
[15] J. Amaré, et al., The ANAIS-112 experiment at the Canfranc Underground Laboratory, in: 15th International Conference on Topics in Astroparticle and Underground Physics (TAUP 2017) Sudbury, Ontario, Canada, July 24-28, 2017, 2017. arXiv:1710.03837

[16] M. Antonello, et al., The SABRE project and the SABRE PoP, arXiv: 1806.09340

[17] S. Agostinelli, et al., Geant4 - a simulation toolkit, Nuclear Instruments and Methods in Physics Research Section A: Accelerators, Spectrometers, Detectors and Associated Equipment 506 (3) (2003) 250 - 303. doi: http://dx.doi.org/10.1016/S0168-9002(03)01368-8

[18] J. Allison, et al., Geant4 developments and applications, IEEE Trans. Nucl. Sci. 53 (2006) 270. doi: 10.1109/TNS. 2006.869826

[19] J. Allison, et al., Recent developments in Geant4, Nuclear Instruments and Methods in Physics Research Section A: Accelerators, Spectrometers, Detectors and Associated Equipment 835 (2016) 186 - 225. doi: https://doi.org/10.1016/j.nima.2016.06.125

[20] V. N. Ivanchenko, et al., Geant4 models for simulation of multiple scattering, J. Phys. Conf. Ser. 219 (2010) 032045. doi:10.1088/1742-6596/219/3/032045

[21] D. Cullen, J. Hubbell, L. Kissel, The Evaluated Photon Data Library, Report UCRL-50400, vol. 6 (1997).

[22] F. Salvat, J. Fernandez-Varea, J. Sempau, Penelope 2008: A Code System for Monte Carlo Simulation of Electron and Photon Transport, Report 6416, OECDNEA, Issy-les-Molineaux, France (2009).

[23] PNNL, private communication.

[24] I. J. Arnquist, E. W. Hoppe, The quick and ultrasensitive determination of $\mathrm{K}$ in $\mathrm{NaI}$ using inductively coupled plasma mass spectrometry, Nuclear Instruments and Methods in Physics Research Section A: Accelerators, Spectrometers, Detectors and Associated Equipment 851 (2017) 15 - 19. doi:https://doi.org/10. 1016/j.nima.2017.01.064

[25] Seastar Chemicals, private communication.

[26] R. Bernabei, et al., The DAMA/LIBRA apparatus, Nuclear Instruments and Methods in Physics Research Section A: Accelerators, Spectrometers, Detectors and Associated Equipment 592 (3) (2008) $297-315$. doi: http://dx.doi.org/10.1016/j.nima.2008.04.082

[27] J. Amaré, et al., Assessment of backgrounds of the ANAIS experiment for dark matter direct detection", European Physical Journal C 76 (8) (2016) 429. doi: 10.1140/epjc/s10052-016-4279-2.

[28] P. Adhikari, et al., Background model for the $\mathrm{NaI}(\mathrm{Tl})$ crystals in COSINE-100, EPJC 78 (6) (2018) 490. doi: 10.1140/epjc/s10052-018-5970-2.

[29] J. Amaré, et al., Cosmogenic radionuclide production in $\mathrm{NaI}(\mathrm{Tl})$ crystals, JCAP 1502 (02) (2015) 046. arXiv: 1411.0106 doi:10.1088/1475-7516/2015/02/046.

[30] ACTIVIA Simulation Software, http:// universityof warwick . github . io/ACTIVIA/

[31] M. Laubenstein, HPGe screening at LNGS.

[32] E. Aprile, et al., Lowering the radioactivity of the photomultiplier tubes for the XENON1T dark matter experiment, European Physical Journal C 75 (2015) 546. doi:10.1140/epjc/s10052-015-3657-5

[33] Radiopurity database, www.radiopurity.org

[34] E. Aprile, et al., Material screening and selection for XENON100, Astroparticle Physics 35 (2) (2011) 43 - 49. doi:http://dx.doi.org/10.1016/j. astropartphys.2011.06.001 
[35] C. Alduino, et al., Measurement of the two-neutrino double-beta decay half-life of ${ }^{130} \mathrm{Te}$ with the CUORE-0 experiment, The European Physical Journal C 77 (1) (2017) 13. doi:10.1140/epjc/s10052-016-4498-6

[36] L. Baudis, et al., Cosmogenic activation of xenon and copper, The European Physical Journal C 75 (10) (2015) 485. doi:10.1140/epjc/s10052-015-3711-3

[37] E. Shields, SABRE: A search for dark matter and a test of the DAMA/LIBRA annual-modulation result using thallium-doped sodium-iodide scintillation detectors, Ph.D. Thesis Princeton University.

[38] P. Agnes, et al., The veto system of the DarkSide-50 experiment, JINST P03016. doi:https://doi.org/10. 1088/1748-0221/11/03/P03016

[39] G. Alimonti, et al., The liquid handling systems for the Borexino solar neutrino detector, Nuclear Instruments and Methods in Physics Research Section A: Accelerators, Spectrometers, Detectors and Associated Equipment 609 (1) (2009) 58 - 78. doi:http://dx.doi.org/ 10.1016/j.nima.2009.07.028

[40] W. B. Wilson, et al., SOURCES: a code for calculating $(\alpha, \mathrm{n})$, spontaneous fission, and delayed neutron sources and spectra, Radiation Protection Dosimetry 115 (1-4) (2005) 117-121. arXiv:/oup/backfile/content_public/journal/ rpd/115/1-4/10.1093/rpd/nci260/2/nci260.pdf doi : 10.1093/rpd/nci260 